\title{
Rentabilidad de la caña de azúcar con manejo orgánico y convencional
}

\author{
Profitability of the sugar cane with organic and conventional management
}

\author{
Cinthia Vanesa Ibarra González, Oscar Raúl Mancilla Villa²*, Rubén Darío Guevara Gutiérrez ${ }^{3}$, \\ Omar Hernández Vargas ${ }^{1}$, Carlos Palomera García ${ }^{4}$, Álvaro Can Chulim ${ }^{5}$, José de Jesús Huerta \\ Olague ${ }^{1}$, Héctor Manuel Ortega Escobar ${ }^{6}$, José Luis Olguin López ${ }^{3}$, Jaqueline Paz González ${ }^{7}$
}

\begin{abstract}
RESUMEN
En Jalisco desde hace 30 años se cultiva caña de azúcar en el valle de Autlán - El Grullo - El Limón, representa una de las zonas más productivas en el estado. La variación de presión en el mercado de insumos y del producto final, generan incertidumbre hacia los productores que carecen de información de rentabilidad en esta actividad productiva. El objetivo de esta investigación fue comparar la rentabilidad del cultivo de caña bajo dos tipos de manejo; el orgánico y el convencional. Se realizó un estudio económico-productivo mediante entrevistas a productores ubicados en el valle en el año de 2016, se llevó a cabo un análisis de rentabilidad para una proyección de 5 años bajo 3 escenarios. Los resultados de la relación Beneficio/costo, El Valor Actual Neto y la Tasa Interna de Retorno indican que el manejo orgánico tiende a ser rentable en mayor medida, de acuerdo con los escenarios analizados, al compararlo con el manejo convencional.
\end{abstract}

Palabras clave: Caña de azúcar, manejo orgánico, manejo convencional, rentabilidad.

\section{ABSTRACT}

Jalisco has been growing sugarcane for 30 years in the Autlán Valley - El Grullo - El Limón, representing one of the most productive areas in the state. The change in price in the market of inputs and the final product, generate uncertainty towards producers who lack information on profitability in this productive activity. The objective of this research was to compare the profitability of cane cultivation under two types of management; the organic and the conventional. An economic-productive study was conducted through interviews with producers located in the valley in the year of 2016, a profitability analysis was carried out for a 5-year projection under 3 scenarios. The results of the benefit / cost ratio, the net present value and the internal rate of return indicate that organic management tends to be more profitable, according to the scenarios analyzed, when compared to conventional management.

Key words: Sugar cane, organic management, conventional management, profitability.

\section{Introducción}

La caña de azúcar (Saccharum officinarum L.) es uno de los principales cultivos mas importantes en las regiones tropicales y subtropicales a nivel mundial (Chastel, 1994). Representa el cultivo más importante en la producción de endulzante del mundo. La caña de azúcar se cultiva en más de 130 países, siendo brasil el mayor productor con $28 \%$ del total de la producción; México ocupa el quinto lugar a nivel mundial (LópezVázquez et al., 2016). El azúcar tiene múltiples usos, ya sea domésticos o industriales; se emplea en la industria alimenticia y de bebidas, las mieles vírgenes para la producción de alcohol y ron, levadura, melaza

1 Ingeniería en Recursos Naturales y Agropecuarios. Departamento de Ecología y Recursos Naturales. Centro Universitario de la Costa Sur (CUCSUR). Universidad de Guadalajara. México.

2 Departamento de Producción Agrícola, Universidad de Guadalajara. México.

3 Departamento de Ecología y Recursos Naturales, Universidad de Guadalajara. México.

4 Ciencias de la Salud y Ecología Humana. CUCSUR. Universidad de Guadalajara. México.

5 Universidad Autónoma de Nayarit.

6 Colegio de Postgraduados. Texcoco, México.

7 Departamento de Ciencias Económicas y Administrativas, CUCSUR. Universidad de Guadalajara.

* Autor de correspondencia: oscar.mancilla@academicos.udg.mx

Fecha de Recepción: 05 marzo, 2018.

Fecha de Aceptación: 06 junio, 2018.

DOI: http://dx.doi.org/10.4067/S0718-34292018005000901. Publicado en línea: 25-octubre-2018. 
para la alimentación animal y químicos orgánicos entre otros (Pratt y Pérez, 2002). En México la zona cañera está constituida por 15 estados de los cuales destacan Puebla, Morelos, Chiapas, Veracruz, San Luis Potosí y Jalisco (Jiménez et al., 2004).

Se ha evidenciado que los gastos de operaciones e insumos varían en cuanto al tipo de manejo (convencional, orgánico y alternativo) por las actividades que realizan cada uno, siendo el manejo convencional el que requiere una mayor inversión financiera (USAID, 2010). Bajo éste contexto, en el valle de Autlán - El Grullo, Jalisco, desde hace aproximadamente 30 años el sistema de producción de caña de azúcar ha sido de tipo convencional, se hace uso indiscriminado de agroquímicos (ya sea para mejorar su producción o eliminar plagas y enfermedades) situación que incrementa los costos de producción.

El presente trabajo de investigación presenta información sobre los manejos orgánico y convencional de la producción de caña de azúcar del valle Autlán - El Grullo - El Limón, Jalisco y aporta conocimientos económicos para la implementación de estrategias en el mejoramiento de la producción de este cultivo. La presente investigación se realizó durante los años 2015 y 2016, haciendo una comparación entre la rentabilidad del cultivo de caña bajo dos diferentes tipos de manejo, el orgánico y el convencional.

\section{Materiales y métodos}

El valle Autlán - El Grullo - El Limón (Figura 1) lo integran los municipios del mismo nombre, se ubica al suroeste del Estado de Jalisco (SAGARPA, 2009). La superficie de caña de azúcar cultivada es de 10,027 ha (Álvarez y Pérez, 2004). El valle está considerado dentro del Distrito de Riego 094 Jalisco Sur (Mancilla-Villa et al., 2017).

Existen diferentes métodos para determinar la rentabilidad económica de un proyecto como las expresiones algebraicas siguientes basadas en la teoría

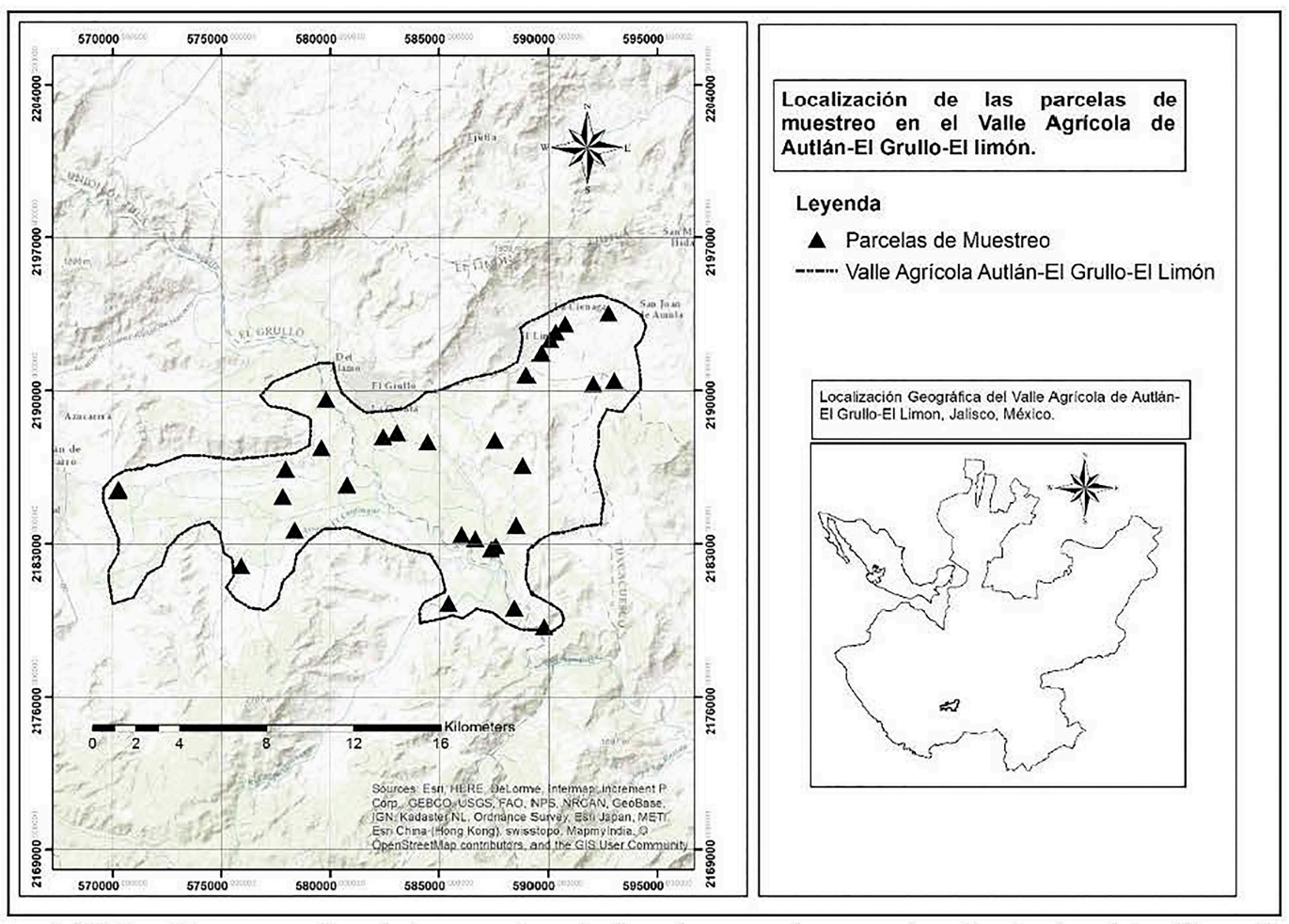

Figura 1. Ubicación geográfica de la zona de estudio y las parcelas muestreadas dentro del valle agrícola. 
económica (Krugman y Wells, 2006; Samuelson y Nordhaus, 2009):

$$
\mathrm{CT}=\mathrm{P}_{\mathrm{X}} \mathrm{X}
$$

Donde:

$\mathrm{CT}=$ Costo total de la producción

$\mathrm{P}_{\mathrm{x}}=$ Precio del insumo o actividad

$\mathrm{X}$ y $\mathrm{Y}=$ Actividad $\mathrm{o}$ insumo

$$
\mathrm{IT}=\mathrm{P}_{\mathrm{y}} \mathrm{Y}
$$

Donde:

IT= Ingreso total (\$ ha-1)

Py=Precio de mercado del cultivo Y (\$ t-1)

$\mathrm{Y}=$ Rendimiento del cultivo (t ha-1).

La rentabilidad es igual a:

\section{Rentabilidad=IT $-\mathrm{CT}$}

Según Retes et al. (2015) la determinación de la rentabilidad de una inversión se hace a través del uso de diversas herramientas financieras como:

Capital de trabajo

Relación Beneficio-Costo (RBC). Para este indicador se utiliza la siguiente ecuación:

$$
R B C=\frac{\sum \text { Ventas }}{\sum \text { Costos }}
$$

Punto de equilibrio

Análisis de sensibilidad

Costo financiero (cálculo de intereses) para este indicador se utiliza la ecuación universal:

$$
\frac{\text { capital } * \text { tasa de interés } * \text { tiempo }}{a \tilde{n} o \text { comercial }}
$$

Para esta investigación se entrevistaron 30 productores (15 con manejo orgánico y 15 con manejo convencional). El análisis de rentabilidad económica de la caña de azúcar para manejo orgánico y manejo convencional se realizó bajo tres escenarios (1. ingresos altos y egresos bajos, 2. promedio, ingresos bajos y 3. egresos altos) mediante los indicadores financieros de valor actual neto (VAN), tasa interna de retorno (TIR) y la relación beneficio-costo $(\mathrm{B} / \mathrm{C})$, utilizando las funciones que refiere Mancilla et al. (2009) las cuales se describen a continuación $(1,2$, y 3$)$ :

$$
B / C=\left[\frac{\sum_{t=0}^{n} \frac{B n}{(1+i)^{n}}}{\sum_{t=0}^{n} \frac{C n}{(1+i)^{n}}}\right] \ldots 1
$$

$$
\begin{gathered}
V A N=\sum_{t=0}^{n} \frac{B n-C n}{(1+i)} \ldots 2 \\
T I R=\sum_{t=0}^{n} \frac{B n-C n}{(1+i)}=0 \ldots 3
\end{gathered}
$$

Donde:

$\mathrm{Bn}=$ beneficios de cada año $(\$)$

$\mathrm{Cn}=$ costos de cada año $(\$)$

$\mathrm{n}=$ número de años

i = tasa de interés (descuento) o actualización (\%)

Los criterios de aceptación de todo proyecto productivo en el aspecto de rentabilidad, de acuerdo con Baca- Urbina (2001) son; que la relacion beneficio/ costo sea mayor a 1 , que el VAN sea positivo y que la TIR sea mayor a la tasa de interés que oferta el mercado para proyectos productivos, que en este caso fue de $10 \%$.

Para obtener información puntual de campo se aplicaron encuestas con preguntas abiertas y cerradas a los productores de manejo orgánico y manejo convencional para estimar los costos y beneficios de la producción, para lo cual se aplicó la metodología mencionada por Malhotra (1997).

Para la obtención del VAN se utilizó como inversión (egresos) los costos que implica la producción de la caña de azúcar y los ingresos (beneficios/ganancias) lo que se obtenga de la venta de la caña de azúcar. Se calculó para un periodo de 5 años, con la tasa de interés de $10 \%$ y se comparó con los ingresos esperados.

Para estimar la TIR se utilizaron los costos de producción los beneficios obtenidos y la tasa de interés del $10 \%$ para un periodo de 5 años. Relación $\mathrm{B} / \mathrm{C}$ se calcularon los beneficios que se obtuvieron durante el periodo de 5 años en relación a los que obtiene actualmente.

Se optó por las funciones VAN, TIR y Relación Costo-Beneficio para determinar la rentabilidad de esta investigación debido a que en el cálculo de estos indicadores se toman en cuenta variables que permiten un análisis más completo de la rentabilidad 
de un proyecto de inversión, como los valores netos actualizados de cada año, la menor tasa de interés que se puede permitir para generar utilidad y el panorama real del valor del proyecto (Baca-Urbina, 2001) y no solo los ingresos y costos totales que manejan otros autores para obtener la utilidad (Retes et al., 2015; Krugman y Wells, 2006; Samuelson y Nordhaus, 2009).

\section{Resultados y discusión}

\section{Inversión}

En la tabla 1 se muestran los costos de inversión para ambos sistemas de manejo los cuales incluyen el costo de instalación del sistema de riego, herramientas y equipo para llevar acabo las prácticas de manejo del cultivo de caña. Se estimó un costo de inversión de \$27, 116.00 ha $^{-1}$. Éste costo de inversión no ésta considerado dentro de los costos de producción pero si se tomaron en cuenta para el análisis de rentabilidad. Se vuelve a invertir hasta que se desgasten o termine su vida útil.
Tabla 1. Costos de inversión para ambos sistemas de manejo los cuales incluyen el costo de instalación del sistema de riego, herramientas y equipo para llevar acabo las prácticas de manejo.

\begin{tabular}{lccc}
\hline Concepto & Costo unitario $(\$)$ & Cantidad & Costo por ha $(\$)$ \\
\hline $\begin{array}{l}\text { Sistema riego } \\
\text { Mochila }\end{array}$ & $23,256.00$ & & $23,256.00$ \\
aspersora & 650.00 & 3 & $1,950.00$ \\
$\begin{array}{l}\text { Mochila para } \\
\text { fetilizar }\end{array}$ & 150.00 & 3 & 450.00 \\
Palas & 120.00 & 2 & 240.00 \\
Machetes & 70.00 & 2 & 140.00 \\
Cazangas & 40.00 & 2 & 80.00 \\
Barra & 200.00 & 2 & 400.00 \\
Botas de Hule & 300.00 & 2 & 600.00 \\
Total & $24,786.00$ & & $27,116.00$ \\
\hline
\end{tabular}

ha :hectárea; \$: pesos Mexicanos.

\section{Costos}

En las tablas 2 y 3 se muestran las actividades y el costo que tienen por ha en siembra y soca, lo que permite diferenciar la siembra de la soca (ultimo retoño de la Caña de azúcar). Se obtuvo un mínimo, promedio y máximo de los costos, debido a que los costos de producción para el productor dependen de los insumos, maquinaria, personal, etc. El costo

Tabla 2. Costos (\$) por ha en manejo orgánico y convencional para siembra.

\begin{tabular}{lcccrrr}
\hline & \multicolumn{1}{c}{ Orgánico } & & \multicolumn{4}{c}{ Convencional } \\
& Siembra & & \multicolumn{4}{c}{ Siembra } \\
Actividad & Mínimo & Promedio & Máximo & Mínimo & Promedio & Máximo \\
\hline Renta & 10,385 & 10,385 & 10,385 & 10,385 & 10,385 & 10,385 \\
Preparación del suelo & 3,750 & 6,764 & 9,350 & 3,750 & 6,764 & 9,350 \\
Siembra & 24,161 & 26,307 & 28,366 & 24,161 & 26,369 & 28,366 \\
Labores culturales & 2,625 & 3,694 & 5,435 & 2,965 & 4,653 & 6,435 \\
Insumos & 19,684 & 30,400 & 45,396 & 27,301 & 31,593 & 31,593 \\
Total & 60,785 & 77,550 & 98,932 & 68,562 & 79,746 & 86,129 \\
\hline
\end{tabular}

Labores culturales: actividades de mantenimiento y cuidado.

Tabla 3. Costos (\$) por ha en manejo orgánico y convencional para socas.

\begin{tabular}{|c|c|c|c|c|c|c|}
\hline Actividad & $\begin{array}{l}\text { Orgánico } \\
\text { Soca } \\
\text { Mínimo }\end{array}$ & Promedio & Máximo & $\begin{array}{l}\text { Convencional } \\
\text { Soca } \\
\text { Mínimo }\end{array}$ & Promedio & Máximo \\
\hline Renta & 10,385 & 10,385 & 10,385 & 10,385 & 10,385 & 10,385 \\
\hline Preparación del suelo & 0 & 0 & 0 & 0 & 0 & 0 \\
\hline Siembra & 0 & 0 & 0 & 0 & 0 & 0 \\
\hline Labores culturales & 3,625 & 5,021 & 7,535 & 4,115 & 6,085 & 8,685 \\
\hline Insumos & 19,684 & 30,400 & 45,396 & 27,301 & 31,593 & 31,593 \\
\hline Total & 33,694 & 45,806 & 63,316 & 41,801 & 48,513 & 51,113 \\
\hline
\end{tabular}

Soca: ultimo retoño de la caña de azúcar. 
de producción de la caña de azúcar por hectárea es diferente cuando se siembra a cuando se tiene socas porque no se realizan las mismas actividades, éstas últimas son menos cuando son socas por ello los costos bajan (Perez et al., 2011).

Las actividades a realizar en ambos manejos cuando es siembra son las que se requieren para la preparación del terreno, plantación, labores culturales e insumos a utilizar; cuando son socas solo se realizan labores culturales e insumos, es por ello que los costos disminuyen de siembra a soca (Cabral y Birbaumer, 2008). Cabe mencionar que los costos de producción varían de acuerdo a la zona productora y de productor a productor (Osorio, 2007) porque aunque tengan el mismo tipo de manejo no aplican los mismos insumos, prefieren omitir alguna actividad o realizarla de manera distinta como el subsuelo simple o doble (costo mínimo o máximo, Tabla 2 y Tabla 3). El costo en el manejo orgánico es más bajo por actividades que se omiten y que se realizan en el manejo convencional como la limpia, junta y quema, además de los fertilizantes que utilizan, debido a que en el manejo orgánico los productores fabrican sus propios fertilizantes reduciendo así sus costos de producción.

El costo total en ambos sistemas de manejo tanto para siembra y soca se obtuvo considerando que todos los productores pagan renta, realizan todas las actividades y aplican todos los insumos considerados que se encontraron en esta investigación, los costos presentados en este trabajo sería lo máximo que podría gastar un productor de caña, ya sea al realizar las actividades más simples (menor costo), al aplicar menos cantidad de insumos o viceversa. En el manejo convencional se tiene un costo promedio para siembra de $\$ 79,746$ y para socas de $\$ 40,617$ (Tabla 7 y Tabla 8 ) en comparación de los costos reportados en el periodo 2013/2015 en siembras con un valor de $\$ 33,899$.

\section{Ingresos}

En la Tabla 4 y Tabla 5 se muestran los beneficios (\$) obtenidos para cada sistema de manejo, se estableció un mínimo, promedio y máximo de lo que se puede obtener de cada sistema de manejo. La diferencia entre los beneficios obtenidos en siembra y soca es el rendimiento obtenido, y la diferencia de los benéficos obtenidos entre un sistema de manejo también se encuentra en el rendimiento.

Se observa que el precio de venta para el año 2015 aumentó a \$502 la tonelada, ya que de acuerdo a lo establecido por Orozco-Hernández et al. (2017) en el año 2014 el precio de venta fue de $\$ 490$, y aun así de acuerdo con los productores el precio de la caña es bajo porque no les deja un beneficio considerable. El benéfico económico es la diferencia entre el ingreso

Tabla 4. Beneficios económicos en manejo orgánico en siembra y soca.

\begin{tabular}{|c|c|c|c|c|c|c|c|c|}
\hline & \multirow[t]{2}{*}{ Beneficio concepto } & \multirow{2}{*}{$\begin{array}{l}\text { Cantidad } \\
\text { (t/ha) } \\
\text { Mín. }\end{array}$} & \multirow[b]{2}{*}{ Prom. } & \multirow[b]{2}{*}{ Máx. } & \multirow{2}{*}{ Precio venta $\$ / t$} & \multicolumn{3}{|c|}{ Beneficio anual (\$) por ha } \\
\hline & & & & & & Mín. & Prom. & Máx. \\
\hline \multirow[t]{2}{*}{ Siembra } & Venta de caña & 167 & 207 & 320 & 502 & 82,328 & 103,914 & 160,640 \\
\hline & Punta de caña (alimento) & 1 & 1 & 1 & 300 & 300 & 300 & 300 \\
\hline Total & & & & & & 82,628 & 104,214 & 160,940 \\
\hline \multirow[t]{2}{*}{ Soca } & Venta de caña & 90 & 139 & 200 & $\$ 502$ & 45,180 & 69,778 & 100,400 \\
\hline & Punta de caña (alimento) & 1 & 1 & 1 & $\$ 300$ & 300 & 300 & 300 \\
\hline Total & & & & & & 45,480 & 70,078 & 100,700 \\
\hline
\end{tabular}

Tabla 5. Beneficios económicos en manejo convencional en siembra y soca.

\begin{tabular}{|c|c|c|c|c|c|c|c|c|}
\hline & \multirow[t]{2}{*}{ Beneficio concepto } & \multirow{2}{*}{$\begin{array}{l}\text { Cantidad } \\
\text { (t/ha) } \\
\text { Mín. }\end{array}$} & \multirow[b]{2}{*}{ Prom. } & \multirow[b]{2}{*}{ Máx. } & \multirow[t]{2}{*}{$\begin{array}{l}\text { Precio } \\
\text { venta } \$ / t\end{array}$} & \multicolumn{3}{|c|}{ Beneficio anual (\$) por ha } \\
\hline & & & & & & Mínimo & Promedio & Máximo \\
\hline \multirow[t]{2}{*}{ Siembra } & Venta de caña & 140 & 179 & 227 & 502 & 70,280 & 89,859 & 113,954 \\
\hline & Punta de caña (alimento) & 1 & 1 & 1 & 300 & 300 & 300 & 300 \\
\hline Total & & & & & & 70,580 & 90,159 & 114,254 \\
\hline \multirow[t]{2}{*}{ Soca } & Venta de caña & 50 & 121 & 185 & $\$ 502$ & 25,100 & 60,742 & 92,870 \\
\hline & Punta de caña (alimento) & 1 & 1 & 1 & $\$ 300$ & 300 & 300 & 300 \\
\hline Total & & & & & & 25,400 & 61,042 & 93,170 \\
\hline
\end{tabular}


recibido por la producción de caña, pero el beneficio disminuye conforme baja el rendimiento y los costos se mantienen iguales o se incrementan (MancillaVilla et al., 2009).

\section{Análisis de rentabilidad}

Se manejaron 3 escenarios de rentabilidad con respecto a cada sistema de manejo como se aprecia en la Tabla 6, el primero, ingresos altos y egresos bajos; el segundo ingresos y egresos promedio y el tercero ingresos bajos y egresos altos, con la finalidad de evaluar los diferentes factores o elementos económicos que pueden propiciarse en la producción agrícola de la caña de azúcar.

En el primer escenario del sistema de manejo orgánico se necesita un costo de inversión de $\$ 87,901.00$ y se obtiene un ingreso el primer año de $\$ 160,40.00$; el costo de producción para el segundo ciclo será de $\$ 35,648$, el costo de producción disminuye, porque ya no se hacen gastos de inversión y se omiten algunas actividades; sin embargo, para el segundo año los ingresos bajan debido a que el rendimiento disminuye. En los siguientes años de proyección, la producción de caña se mantiene y en los dos últimos años de evaluación disminuye.

Los costos e ingresos a partir del segundo año disminuyen. Conforme se avanza en el tiempo, el rendimiento de caña es menor; en ese sentido los costos de producción serán menores, toda vez que para establecer el cultivo de caña se requieren más insumos, mano de obra y otros costos, que a partir del segundo año se prescinde de ellos puesto que se produce con soca. El flujo de efectivo se calculó con una tasa de interés de 10\% (Tabla 7).

En el segundo escenario del sistema de manejo orgánico se necesita un costo de inversión de $\$ 104,666.00$ y se obtiene un ingreso el primer año de $\$ 104,214.00$; el costo de producción para el segundo ciclo será de $\$ 48,462.74$ y los ingresos de $\$ 74,142.52$. Al final del periodo de 5 años los costos de producción serán de $\$ 60,722.61$ con un ingreso de $\$ 87,806.02$ y un flujo de efectivo de 27,083.41 (Tabla 8).

En el tercer escenario del sistema de manejo orgánico se necesita un costo de inversión de $\$ 126,048.00$ se obtiene un ingreso el primer año de

Tabla 6. Ingresos altos y egresos bajos del sistema de manejo orgánico en caña de azúcar.

\begin{tabular}{lcccccc}
\hline Año & $\begin{array}{c}\text { Ingresos } \\
(\$)\end{array}$ & $\begin{array}{c}\text { Costos } \\
(\$)\end{array}$ & Flujo de efectivo & $\begin{array}{c}\text { Tasa } \\
(1+\mathrm{t})-\mathrm{n}\end{array}$ & $\begin{array}{c}\text { Ingresos } \\
\text { actualizados } \\
(\$)\end{array}$ & $\begin{array}{c}\text { Egresos } \\
\text { actualizados } \\
(\$)\end{array}$ \\
\hline 0 & $\$-$ & $87,901.00$ & $-87,901.00$ & 1.00000 & $\$-$ & $87,901.00$ \\
1 & 160,940 & $35,648.25$ & $125,291.75$ & 0.90909 & $146,309.09$ & $32,407.50$ \\
2 & 106,540 & $37,715.85$ & $68,824.75$ & 0.82645 & $88,050.08$ & $31,170.12$ \\
3 & 112,719 & $39,903.37$ & $72,815.98$ & 0.75131 & $84,687.72$ & $29,979.99$ \\
4 & 119,257 & $42,217.76$ & $77,039.31$ & 0.68301 & $81,454.18$ & $28,835.30$ \\
5 & 126,173 & $44,666.39$ & $81,507.59$ & 0.62092 & $78,344.11$ & $27,734.31$ \\
Total & $625,631.00$ & $288,052.00$ & $337,578.38$ & & $478,845.19$ & $238,028.23$ \\
\hline
\end{tabular}

Tabla 7. Ingresos y egresos promedio del sistema de manejo orgánico en caña de azúcar.

\begin{tabular}{lcccccc}
\hline Año & $\begin{array}{c}\text { Ingresos } \\
(\$)\end{array}$ & $\begin{array}{c}\text { Costos } \\
(\$)\end{array}$ & $\begin{array}{c}\text { Flujo de } \\
\text { efectivo } \\
(\$)\end{array}$ & $\begin{array}{c}\text { Tasa } \\
(1+\mathrm{t})-\mathrm{n}\end{array}$ & $\begin{array}{c}\text { Ingresos } \\
\text { actualizados } \\
(\$)\end{array}$ & $\begin{array}{c}\text { Egresos } \\
\text { actualizados } \\
(\$)\end{array}$ \\
\hline 0 & $\$-$ & $104,666.00$ & $-104,666.00$ & 1.00000 & $\$-$ & $104,666.00$ \\
1 & $104,214.00$ & $48,462.74$ & $55,751.26$ & 0.90909 & $94,740.00$ & $44,057.04$ \\
2 & $74,142.52$ & $51,273.57$ & $22,868.95$ & 0.82645 & $61,274.81$ & $42,374.85$ \\
3 & $78,442.78$ & $54,247.43$ & $24,195.35$ & 0.75131 & $58,935.22$ & $40,756.90$ \\
4 & $82,992.46$ & $57,393.78$ & $25,598.68$ & 0.68301 & $56,684.97$ & $39,200.72$ \\
5 & $87,806.02$ & $60,722.61$ & $27,083.41$ & 0.62092 & $54,520.63$ & $37,703.96$ \\
Total & $427,597.78$ & $376,766.13$ & $50,831.65$ & & $326,155.63$ & $308,759.47$ \\
\hline
\end{tabular}

Tabla 8. Ingresos bajos y egresos altos del sistema de manejo orgánico en caña de azúcar.

\begin{tabular}{lcccccc}
\hline Año & $\begin{array}{c}\text { Ingresos } \\
(\$)\end{array}$ & $\begin{array}{c}\text { Costos } \\
(\$)\end{array}$ & $\begin{array}{c}\text { Flujo de } \\
\text { efectivo }(\$)\end{array}$ & $\begin{array}{l}\text { Tasa } \\
(1+\mathrm{t})-\mathrm{n}\end{array}$ & $\begin{array}{c}\text { Ingreso } \\
\text { actualizado }(\$)\end{array}$ & $\begin{array}{c}\text { Egreso } \\
\text { actualizado }(\$)\end{array}$ \\
\hline 0 & $\$-$ & $126,048.00$ & $-126,048.00$ & 1.00000 & $\$-$ & $126,048.00$ \\
1 & $82,628.00$ & $66,988.32$ & $15,639.68$ & 0.90909 & $75,116.36$ & $60,898.47$ \\
2 & $48,117.84$ & $70,873.64$ & $-22,755.80$ & 0.82645 & $39,766.81$ & $58,573.26$ \\
3 & $50,908.67$ & $74,984.31$ & $-24,075.64$ & 0.75131 & $38,248.44$ & $56,336.82$ \\
4 & $53,861.37$ & $79,333.40$ & $-25,472.03$ & 0.68301 & $36,788.04$ & $54,185.78$ \\
5 & $56,985.32$ & $83,934.73$ & $-26,949.41$ & 0.62092 & $35,383.40$ & $52,116.86$ \\
Total & $292,501.20$ & $502,162.40$ & $-209,661.20$ & & $225,303.05$ & $408,159.19$ \\
\hline
\end{tabular}


$\$ 82,628.00$, el costo de producción para el segundo ciclo será de $\$ 66,998.32$ y los ingresos de $\$ 48,117.84$ (Tabla 9).

En el primer escenario del sistema de manejo convencional se requiere un costo de inversión de $\$ 95,678.00$ y se obtiene un ingreso el primer año de $\$ 114,254.00$, el costo de producción para el segundo ciclo será de $\$ 44,225.45$ y los ingresos de $\$ 98,573.86$ (Tabla 10).

En el segundo escenario del sistema de manejo convencional se requiere un costo de inversión de $\$ 106,832.00$ y se obtiene un ingreso el primer año de $\$ 90,159.00$, el costo de producción para el segundo ciclo será de $\$ 51,326.75$ y los ingresos de $\$ 64,582.43$ (Tabla 11).

El tercer escenario del sistema de manejo convencional se requiere un costo de inversión de $\$ 113,245.00$ y se obtiene un ingreso el primer año de $\$ 70,580.00$, el costo de producción para el segundo ciclo será de $\$ 54,077.55$ y los ingresos de $\$ 26,873.20$. El flujo de efectivo total fue negativo porque es mayor el costo que el ingreso, ya que no se recuperan los costos de inversión con una diferencia negativa entre ingresos y egresos de $\$ 229,072.55$.

El VAN, TIR y B/C son los indicadores de rentabilidad y los que permiten tomar la decisión de llevar a cabo el proyecto o no (Tabla 12), éstos indican si es rentable en éste caso el sistema de producción de caña de azúcar (Baca-Urbina, 2001).

El VAN para el manejo orgánico en el tercer escenario fue negativo, igual que en el manejo convencional en el segundo y tercer escenario resultaron negativos; en el primer y segundo escenario del manejo orgánico fueron positivos, al igual que el primer escenario del manejo convencional y esto representa la ganancia obtenida para el periodo de evaluación, de acuerdo con Mancilla-Villa (2009).

La TIR en el primer escenario con manejo orgánico es de $111.80 \%$, en el segundo escenario es de $17.39 \%$, en el primer escenario con manejo convencional se obtuvo $57.05 \%$ y en el segundo $-9.34 \%$.

Tabla 9. Ingresos altos y egresos bajos del sistema de manejo convencional en caña de azúcar.

\begin{tabular}{lrrrrrr}
\hline Año & $\begin{array}{c}\text { Ingresos } \\
\$\end{array}$ & \multicolumn{1}{c}{$\begin{array}{c}\text { Costos } \\
\$\end{array}$} & $\begin{array}{c}\text { Flujo de } \\
\text { efectivo } \$\end{array}$ & $\begin{array}{c}\text { Tasa } \\
(1+\mathrm{t})-\mathrm{n}\end{array}$ & $\begin{array}{c}\text { Ingreso } \\
\text { actualizado }(\$)\end{array}$ & $\begin{array}{c}\text { Egreso } \\
\text { actualizado }(\$)\end{array}$ \\
\hline 0 & \multicolumn{1}{c}{$\$-$} & $95,678.00$ & $-95,678.00$ & 1.00000 & $\$-$ & $95,678.00$ \\
1 & $114,254.00$ & $44,225.45$ & $70,028.55$ & 0.90909 & $103,867.27$ & $40,204.95$ \\
2 & $98,573.86$ & $46,790.52$ & $51,783.34$ & 0.82645 & $81,466.00$ & $38,669.85$ \\
3 & $104,291.14$ & $49,504.37$ & $54,786.77$ & 0.75131 & $78,355.48$ & $37,193.37$ \\
4 & $110,340.02$ & $52,375.62$ & $57,964.40$ & 0.68301 & $75,363.72$ & $35,773.25$ \\
5 & $116,739.74$ & $55,413.40$ & $61,326.34$ & 0.62092 & $72,486.19$ & $34,407.36$ \\
Total & $544,198.76$ & $343,987.36$ & $200,211.40$ & & $411,538.66$ & $281,926.79$ \\
\hline
\end{tabular}

Tabla 10. Ingresos y egresos promedio del sistema de manejo convencional en caña de azúcar.

\begin{tabular}{ccccccc}
\hline Año & $\begin{array}{c}\text { Ingresos } \\
(\$)\end{array}$ & \multicolumn{1}{c}{$\begin{array}{c}\text { Costos } \\
(\$)\end{array}$} & $\begin{array}{c}\text { Flujo de } \\
\text { efectivo }(\$)\end{array}$ & $\begin{array}{c}\text { Tasa } \\
(1+\mathrm{t})-\mathrm{n}\end{array}$ & $\begin{array}{c}\text { Ingreso } \\
\text { actualizado }(\$)\end{array}$ & $\begin{array}{c}\text { Egreso } \\
\text { actualizado }(\$)\end{array}$ \\
\hline 0 & $\$-$ & $106,832.00$ & $-106,832.00$ & 1.00000 & $\$-$ & $106,832.00$ \\
1 & $90,159.00$ & $51,326.75$ & $38,832.25$ & 0.90909 & $81,962.73$ & $46,660.68$ \\
2 & $64,582.43$ & $54,303.70$ & $10,278.73$ & 0.82645 & $53,373.91$ & $44,879.09$ \\
3 & $68,328.21$ & $57,453.31$ & $10,874.90$ & 0.75131 & $51,336.00$ & $43,165.52$ \\
4 & $72,291.24$ & $60,785.60$ & $11,505.64$ & 0.68301 & $49,375.89$ & $41,517.38$ \\
5 & $76,484.30$ & $64,311.16$ & $12,173.14$ & 0.62092 & $47,490.73$ & $39,932.17$ \\
Total & $371,845.18$ & $395,012.52$ & $-23,167.34$ & & $283,539.25$ & $322,986.85$ \\
\hline
\end{tabular}

Tabla 11. Ingresos bajos y egresos altos del sistema de manejo convencional en caña de azúcar.

\begin{tabular}{lcccccc}
\hline Año & $\begin{array}{c}\text { Ingresos } \\
(\$)\end{array}$ & $\begin{array}{c}\text { Costos } \\
(\$)\end{array}$ & $\begin{array}{c}\text { Flujo de efectivo } \\
(\$)\end{array}$ & $\begin{array}{c}\text { Tasa } \\
(1+\mathrm{t})-\mathrm{n}\end{array}$ & $\begin{array}{c}\text { Ingreso } \\
\text { actualizadso }(\$)\end{array}$ & $\begin{array}{c}\text { Egreso actualizado } \\
(\$)\end{array}$ \\
\hline 0 & $\$-$ & $113,245.00$ & $-113,245.00$ & 1.00000 & $\$-$ & $113,245.00$ \\
1 & $70,580.00$ & $54,077.55$ & $16,502.45$ & 0.90909 & $64,163.64$ & $49,161.41$ \\
2 & $26,873.20$ & $57,214.04$ & $-30,340.84$ & 0.82645 & $22,209.26$ & $47,284.33$ \\
3 & $28,431.84$ & $60,532.45$ & $-32,100.61$ & 0.75131 & $21,361.26$ & $45,478.93$ \\
4 & $30,083.88$ & $64,043.33$ & $-33,959.45$ & 0.68301 & $20,547.69$ & $43,742.46$ \\
5 & $31,828.74$ & $67,757.84$ & $-35,929.10$ & 0.62092 & $19,763.14$ & $42,072.29$ \\
Total & $187,797.66$ & $416,870.21$ & $-229,072.55$ & & $148,044.99$ & $340,984.41$ \\
\hline
\end{tabular}


Tabla 12. Análisis de rentabilidad del sistema de manejo orgánico y convencional en la producción de caña de azúcar.

\begin{tabular}{lcccccc}
\hline Indicadores & $\begin{array}{c}\text { Orgánico } \\
\text { Ingresos } \\
\text { altos, } \\
\text { egresos } \\
\text { bajos } \$\end{array}$ & Promedio & $\begin{array}{c}\text { Ingresos } \\
\text { bajos, } \\
\text { egresos } \\
\text { altos }\end{array}$ & $\begin{array}{c}\text { Convencional } \\
\text { Ingresos } \\
\text { altos, } \\
\text { egresos } \\
\text { bajos }\end{array}$ & $\begin{array}{c}\text { Promedio } \\
\text { Ingresos } \\
\text { bajos, } \\
\text { egresos } \\
\text { altos }\end{array}$ \\
\hline VAN & $240,816.96$ & $17,396.16$ & $-182,856.14$ & $129,611.88$ & $-39,447.59$ & $-192,939.42$ \\
TIR & $111.80 \%$ & $17.39 \%$ & & $57.05 \%$ & $-9.34 \%$ & \\
B/C & 2.01 & 1.06 & 0.55 & 1.46 & 0.88 & 0.43 \\
\hline
\end{tabular}

La relación $\mathrm{B} / \mathrm{C}$ con base en manejo orgánico, se obtuvo que en los dos primeros escenarios son rentables, en el manejo convencional solo para el primer escenario es rentable; este indicador recomienda que el manejo orgánico tiende a ser mayor en términos de rentabilidad; con base en los resultados obtenidos en este estudio; se recomienda a los productores de caña que se implemente el manejo orgánico en la producción de este cultivo, tanto desde el punto de vista de rendimiento como desde el aspecto económico.

En un estudio de rentabilidad en el valle de Autlán - El Grullo para manejo alternativo (orgánico) y convencional en caña de azúcar, se obtuvo un VAN promedio para el manejo orgánico de $\$ 179,128$; una TIR de $132 \%$ y una relación B/C de 2.56; en manejo convencional obtuvo un VAN de $\$ 168,661$, una TIR de $117 \%$ y una relación B/C de 2.34 (USAID, 2010); valores muy diferentes a los obtenidos en este estudio. Ésto se debe a que los costos e ingresos obtenidos por USAID son diferentes debido a que en el manejó los costos para siembra de $\$ 34,023$ y socas de $\$ 22,101$, además el rendimiento obtenido fue de 97.20 t/ha en orgánico y $107.53 \mathrm{t} / \mathrm{ha}$ en convencional a un precio de $\$ 680$ /t, y que el precio de la caña de azúcar en este año (2015) se encontró en $\$ 502$, con una diferencia de \$178. Además USAID encontró que en el manejo orgánico los costos son menores que en el manejo convencional; en eso coinciden ambos trabajos, pero USAID menciona que el manejo convencional percibe mayores beneficios económicos porque obtiene mayor rendimiento que el orgánico, en contraste en el presente trabajo los resultados se observan de manera inversa, por lo que el manejo convencional resultó rentable en menor medida que el orgánico.

En el Estado de Veracruz se realizó un análisis de rentabilidad para el Ingenio Tres Valles, la relación de $\mathrm{B} / \mathrm{C}$ fue de $1.27 \%$; el resultado fue que la rentabilidad es de media a baja, comparada con otras regiones cañeras del país; porque dejaría utilidades de $\$ 3,800$ a $\$ 24,000$ ha $^{-1}$ (FIRA, 2007). En otro estudio se encontró que en el área de abasto de Ingenio Central Progreso, la producción de caña de azúcar no genera ganancias ni pérdidas (VAN=0) (Baca-Urbina, 2001).

En Puebla la producción de caña de azúcar para el Ingenio de Atencingo, obtuvo una relación de B/C de 1.23; la rentabilidad es considerada alta debido a la obtención de utilidades de $\$ 10,000$ a $\$ 55,000$ / ha (FIRA, 2006). En Paraguay la rentabilidad de la caña de azúcar orgánica para un periodo de 5 años no es rentable, ya que el rendimiento de la caña es menor y obtiene menores ganancias, comparadas con lo obtenido bajo un sistema convencional (Friedmann y Penner, 2010).

\section{Conclusión}

En el escenario ingresos altos, egresos bajos y promedio del manejo orgánico el VAN y la TIR fueron positivos y en el manejo convencional en los mismos dos escenarios los indicadores resultaron negativos, lo que indica no ser rentable, mientras que el primer manejo arrojó resultados de rentabilidad.

En el primer escenario el VAN para el manejo orgánico fue de $\$ 240,816.96$ y para el convencional de $\$ 129,611.88$; la TIR fue de $111.80 \%$ y $57.05 \%$ y la relación B/C de 2.01 y de 1.46; en el segundo escenario el VAN en el manejo orgánico fue de \$17,396.16 y en el convencional de $-\$ 39,447.59$; la TIR de $17.39 \%$ y $-9.34 \%$ y la relación $\mathrm{B} / \mathrm{C}$ de 1.6 y de 0.88 ; el tercer escenario resulto no rentable en ambos sistemas.

Conforme a los tres escenarios planteados en el análisis de rentabilidad se concluye que el manejo orgánico tiende a generar mayor rentabilidad que el manejo convencional, no obstante, al utilizar la lógica capitalista se esperaba que el manejo convencional fuera rentable y el orgánico no. A cada productor se le presentan escenarios diferentes en los distintos 
manejos, es decir, la rentabilidad en la producción de caña, dependerá de las variaciones en los costos de producción y del precio del producto en el mercado.
Los distintos escenarios y los manejos diferenciados en el cultivo agrícola, definirán la rentabilidad con respecto a la producción de caña de azúcar.

\section{Literatura citada}

Agencia del Gobierno de los Estados Unidos para el Desarrollo Internacional (USAID).

2010. Azúcar Orgánica; Potencial de Negocios. Informe Especial. Paraguay. 90 p.

Álvares, C. M., y Pérez, D. J. F.

2004. Resultados del monitoreo para diagnóstico y control de poblaciones de barrenadores del tallo en caña de azúcar en los municipios: El Grullo - Autlán - El Limón en Jalisco, primer año. INIFAP. 23p.

Baca U. G.

2001. Evaluación de Proyectos $4^{a}$ Edición. McGraw-Hill. 404 p.

Cabral, J., y Birbaumer, G.

2008. La producción de caña dulce orgánica en rotación con otros cultivos orgánicos en pequeñas fincas de la región centro de Paraguay. Azucarera Paraguaya S. A., y Cooperación Técnica Alemana: 25-34.

Chastel J.M.

1994. Le sucre et ses marchés. Agriculture et développmente, 4:4-11.

Fideicomisos Instituidos en Relación con la Agricultura.

2006. Caña de azúcar, Ingenio Atencingo: Análisis de rentabilidad zafra 2005-2006 y proyección de la rentabilidad zafra 20062007. Puebla, México: 12-24.

Fideicomisos Instituidos en Relación con la Agricultura. 2007. Caña de azúcar, Ingenio Tres Valles: Análisis de rentabilidad zafra 2005-2006 y proyección de la rentabilidad zafra 20072008. Veracruz, México: 14-30.

Fridmann, A., y Penner, R.

2010. Azúcar orgánica: potencial de negocios. (USAID/Paraguay (346): 55-59.

Jiménez, C. A., Vargas, T. V., Salinas, C. W. E., Aguirre, B. M J., y Rodríguez, C. D.

2004. Aptitud agroecológica del cultivo de la caña de azúcar en el sur de Tamaulipas, México. Investigaciones Geográficas $M x$, (53): 58-74.

Krugman Paul, y Robin Wells.

2006. Introducción a la Economía, microeconomía, Reverte, Barcelona España. 537 p.
López, V.J.J., Valdez, B.A., Silva, R.H.V., Flores, R.C., Rangel, O.C.A. 2016. Evaluación a la escaldadura (Xanthomonas albilineans (Ashby) Dowson) de la hoja de variedades de caña de azúcar (Saccharum spp.). Agroproductividad, 9 (3): 48-53.

Malhotra, N. K.

1997. Investigación de mercados: Un enfoque práctico. Quinta edición. Pearson Educación. México. 920 p.

Mancilla V. O.R., Oropeza M. J.L. y Martínez M. M.R.

2009. Evaluación de terrazas de banco para plantaciones forestales comerciales. Ciencia Forestal en México. 34 (105): 99-118.

Mancilla, V.O.R., Fregoso, Z.B.E., Hueso, G.E.J., Guevara, G.R.D., Palomera, G.C., Olguín, L.J.L., Ortega, E.H.M., Medina, V.E.K. y Flores, M.H.

2017. Concentración iónica y metales pesados en el agua de riego de la cuenca del río Ayuquila-Tuxcacuesco-Armería. IDESIA, 35 (3): 115-123.

Orozco, H.M.E., García, F.B., Álvarez, A.G., y Miereles L.P. 2017. Tendencias del sector Agrícola, Estado de México. Quivera, 19 (I): 99-121.

Osorio, C. G.

2007. Manual técnico: Buenas prácticas agrícolas-BPA-y buenas prácticas de manufactura-BPM- en la producción de caña y panela. CORPOICA, MANA y FAO: 63-72.

Perez, D., Paredes, V., Rodriguez, G., Scandaliaris, J., y Fandos, C. 2011. Análisis economico del cultivo de caña de azúcar en la campaña 2010/11. Reporte Agroindustrial: Estadísticas y márgenes de cultivos tucumanos. Boletín Tucumán, (6): 2-5.

Pratt, L., y Pérez, J. M.

1997. Industria azucarera en El Salvador: Análisis de sostenibilidad. CEN 631: 2-11.

Samuelson, P. A., y W. D. Nordhaus. 2009. Economía, 19ª Edición, McGraw-Hill, Madrid, España. $744 \mathrm{p}$.

Secretaría de Agricultura, Ganadería, Desarrollo Rural, Pesca y Alimentación.

2009. Un Horizonte Acerca del Mercado Agropecuario. Claridades agropecuarias, 193: 4-6. 
